\title{
GESTIÓN DEL CONOCIMIENTO EN PYMES Y DESEMPEÑO COMPETITIVO ${ }^{1}$
}

\author{
Salvador Estrada ${ }^{2}$
}

Gabriela Dutrénit ${ }^{3}$

\begin{abstract}
Resumen: Este trabajo analiza la gestión del conocimiento en pequeñas y medianas empresas a partir de la perspectiva de la medición del capital intelectual. La evidencia empírica muestra que los recursos intangibles favorecen el desempeño competitivo. El objetivo es identificar el capital intelectual incorporado en prácticas de gestión del conocimiento y analizar su influencia en el desempeño competitivo. La fuente de información es una encuesta en la industria mexicana de maquinados industriales. Mediante análisis multivariados se identifican grupos de prácticas y se estima su influencia sobre el desempeño competitivo. Los resultados muestran que el capital humano está asociado al cumplimiento de una amplia gama de objetivos competitivos, mientras que el capital tecnológico y el relacional tienen efectos selectivos.
\end{abstract}

Palabras clave: capital intelectual, recursos intangibles, análisis multivariado, maquinados, México.

\begin{abstract}
This paper analyzes the Knowledge Management Practices (KMP) in small and medium enterprises from an intellectual capital measurement approach. The empirical review shows than intangibles resources enhance the competitive performance. The main purpose of this research focuses on identifying the intellectual capital embodied on KMP and analyzing its influence on competitive performance. The source of information relies on a survey of the machine shop Mexican industry. Sets of KMP are revealed through the use of multivariate analysis and its influence on competitive performance is assessed. The results show that human capital is related to a wide range of competitive goals while the technological and the relational capital have a more narrow effect on those goals.
\end{abstract}

Key words: intellectual capital, intangible resources, multivariate analysis, machine shop, Mexico.

\footnotetext{
${ }^{1}$ Este trabajo se desarrolló en el marco de la estancia posdoctoral en la Universidad Autónoma Metropolitana-Xochimilco, financiada por CONACYT.

${ }^{2}$ Universidad de Guanajuato, salvador.estrada@gmail.com.

${ }^{3}$ Universidad Autónoma Metropolitana-Xochimilco, gdutrenit@laneta.apc.org.
} 


\section{INTRODUCCIÓN}

El nuevo paradigma de desarrollo y competitividad está basado en la explotación de la información, como materia prima, y del conocimiento, como recurso estratégico (Castells 2000). Tal modo de producción da lugar a una nueva economía, la cual está caracterizada por una creciente globalización de los intercambios comerciales y los flujos de inversión, una intensificación de la competencia internacional, patrones de consumo y distribución cada vez más sofisticados, un alto grado complejidad de los sistemas productivos así como un incremento en las competencias tecnológicas de las empresas y el acortamiento del ciclo de vida de los productos. Este nuevo entorno está transformando las reglas del juego de la competencia, por lo que las empresas están encontrando grandes dificultades para mantener sus ventajas competitivas.

Ante los nuevos retos habría que repensar las estrategias organizacionales. Se puede añadir valor a una organización mediante la gestión del conocimiento, esto es, vincular a la estrategia $y$ funcionamiento de la empresa las prácticas de creación, difusión y apropiación de conocimiento. La estructura de la empresa debe modificarse de tal suerte que sus diversas funciones puedan participar en redes $\mathrm{y}$, mediante la adopción de diversas prácticas, facultarse de capacidades para extraer la información de dichas redes y transformarla en conocimiento valioso para la toma de decisiones (Prusak 1997).

El estudio de la gestión de conocimiento en las organizaciones se da a través de tres vertientes: los procesos generadores de conocimiento, los procesos de aprendizaje, transmisión $\mathrm{y}$ difusión de conocimiento y la medición de los activos intangibles (Bueno y Morcillo 2002). El presente artículo construye sobre este último enfoque $y$ tiene como objetivo identificar el capital intelectual incorporado en prácticas de gestión, analizar la influencia del capital intelectual en el desempeño competitivo, definido por la importancia en el logro de objetivos de innovación, y discriminar entre estos objetivos de acuerdo a su dependencia en la localización espacial.

La fuente de información es una encuesta levantada en la industria de maquinados industriales en dos localidades mexicanas entre 2005 y 2006. La información se analiza mediante un análisis multivariado para identificar grupos de prácticas empresariales que expresan diferentes activos intangibles. Se estima su influencia sobre el desempeño competitivo a través de un conjunto de regresiones logísticas que asocian el cumplimiento de diversos objetivos innovadores a los recursos intangibles.

\section{LOS RECURSOS INTANGIBLES Y SU EFECTO SOBRE EL DESEMPEÑO COMPETITIVO}

El análisis de los recursos intangibles ha sido abordado por la literatura sobre estrategia de la firma. En particular, el enfoque de la teoría de recursos y capacidades, bajo el argumento de que los recursos organizacionales están detrás de la ventaja competitiva, estudia las formas en que se aplican $y$ se combinan, dinámicamente, estos recursos para producir dicha ventaja (Wernerfelt 1984, Barney 1991 Grant, 1991; Amit y Schoemaker, 1993; Peteraf, 1993; Collis y Montgomery, 1995).

Los recursos organizacionales incluyen todos los activos controlados por la empresa y que le habilitan para 
implementar estrategias que mejoren su eficiencia y efectividad (Barney 1991). Los recursos intangibles son altamente valorados por el mercado ${ }^{4}$. En los procesos de creación de valor, recursos intangibles, tales como la creatividad, el talento o una perspectiva innovadora, pueden generar ventajas competitivas sostenibles dado que son únicos, difíciles de imitar, de naturaleza tácita y complejos (Dierickx y Cool 1989). Así se asocia el éxito competitivo a la disponibilidad y acumulación de recursos intangibles.

La viabilidad y éxito de una estrategia depende de los recursos intangibles que ésta pueda movilizar (Itami y Roehl 1987). Pero, la disponibilidad, acumulación y evolución de dichos recursos también está influida por el conjunto de estrategias implementadas.

Así como a nivel macro el residual de Solow puede explicar las diferencias en la explotación de los factores, a nivel de la firma un conjunto de recursos intangibles puede explicar las diferencias entre el valor contable y el valor de mercado. A este plus en la valoración de los recursos financieros y físicos se le ha denominado Capital Intelectual, y está constituido, básicamente, por el aprendizaje organizacional y el desarrollo de relaciones "valiosas" para la empresa.

Es una regla mencionar que todo aquello que se pretende administrar es menester medirlo. El capital intelectual no es la excepción. Así, se está llegando a un consenso sobre el tipo de actividades y recursos que comprende el capital intelectual (Bontis et al. 2000, Sánchez et al. 2000). En general se acepta que está constituido por tres tipos de capital: el humano, el relacional

\footnotetext{
${ }^{4}$ La valoración de los accionistas no coincide con el valor contable de la empresa. Esta diferencia se ha asociado con los recursos intangibles (Sveiby 1997).

${ }^{5}$ Le permiten a la firma afrontar fallas de mercado y reducir la incertidumbre del entorno.
}

y el estructural, los cuales son depósitos de conocimiento ${ }^{6}$.

El capital humano tiene que ver con el nivel cognitivo de los individuos. Su gestión radica en propiciar un ambiente favorable para su externalización, así como expandirlo y restaurarlo a través de las inversiones en entrenamiento y capacitación, así como con el sistema de incentivos (Bontis 2002). El capital relacional está incorporado en las relaciones externas de la firma. Su gestión se centra en mantener flujos de información con los agentes externos y su adecuada distribución al interior de la organización (Roos et al. 1998). El capital estructural se refiere a los depósitos internos de conocimiento tanto físicos como virtuales, esto es, abarca desde las bases de datos, manuales, patentes o marcas hasta las rutinas, normas, capacidades, sistemas y cultura establecidas en la organización. $\mathrm{Su}$ gestión se basa en el aprendizaje organizacional, en la gestión de los sistemas y tecnologías de información, así como de la gestión patrimonial de los recursos tecnológicos (Bontis et al. 2000).

El capital estructural se asocia a una serie de soportes que permiten compartir conocimiento, contribuyen a mejorar la eficiencia de la organización, aceleran los flujos de información y transforman el conocimiento en ganancias. Otra característica es que son propiedad de la organización $\mathrm{y}$ responden a las necesidades del mercado. Para facilitar su identificación, Bueno (2002) propone subdividirlo en aspectos relacionados con la organización y la toma de decisiones, por un lado, y conocimientos tecnológicos, por otro. Así, el capital estructural se divide en capital

\footnotetext{
${ }^{6}$ Se trata de bases de conocimiento estructuradas y distribuidas entre un grupo de personas, como puede ser la memoria de grupo (Cabrera Izquierdo y Rincón Hércules 2001).
} 
organizacional y capital tecnológico, respectivamente. El capital organizacional se define como: el conjunto de intangibles que estructuran $y$ desarrollan de manera eficaz $y$ eficiente la actividad de la organización. Sus elementos constitutivos son: la cultura, la estructura, el aprendizaje organizativo y los procesos en que se soporta la actividad productiva de la organización. El capital tecnológico se refiere al conjunto de intangibles de base técnica responsables tanto de la obtención de productos y del desarrollo de procesos como del avance en la base de conocimientos necesarios para desarrollar futuras innovaciones $\mathrm{Se}$ compone del esfuerzo en $\mathrm{I}+\mathrm{D}+\mathrm{i}$, la dotación tecnológica, la propiedad intelectual e industrial y los resultados de la innovación (Bueno 2002).

Diversas corrientes teóricas argumentan que el capital intelectual incide en el desempeño competitivo (García y Navas 2004). Desde la teoría de la administración estratégica, el capital intelectual cumple las condiciones manifiestas de los recursos estratégicos, esto es se trata de una fuente de competencias distintivas (por su especificidad, complejidad, carácter tácito). La teoría de la firma basada en el conocimiento, la cual busca dar luz sobre las relaciones entre las capacidades tecnológicas y el desempeño, sugiere que la gestión del conocimiento puede ser la fuente de un desempeño superior (DeCarolis y Deeds 1999).

El capital intelectual no necesariamente puede manifestarse directamente sobre el desempeño financiero sino sobre el proceso de creación de valor (Lynn 1998), esto es, la forma en que el mercado y los clientes perciben que se da satisfacción a sus necesidades. Existe evidencia empírica que corrobora una asociación positiva entre el gasto de investigación y desarrollo, las patentes o las actividades innovadoras (como variables proxies del stock de recursos intangibles) con respecto al valor de mercado (Griliches 1981; Conolly y Hirschey 1984; Hirschey y Weygandt 1985, Johnson y Pazderka 1993, Pakes 1985)

La dotación heterogénea de intangibles y su diversa naturaleza (conocimientos tácitos y específicos, de difícil imitación, con complementariedades con los conocimientos explícitos, el potencial de codificación) propician que no exista una sola combinación óptima para alcanzar ciertos objetivos específicos, sino que puede haber una diversidad dependiendo de los patrones de aprendizaje tecnológico y del contexto histórico-locacional específico (Dosi 1988, Pisano, 1994; Teng y Cummings, 2002, García y Navas 2004).

La medición del conocimiento y de los recursos intangibles, así como su valoración económica presenta grandes dificultades. Al nivel de la firma se han desarrollado diversos modelos de medición. Parten del reconocimiento de que los activos intensivos en conocimiento (por ejemplo, la información, la experiencia o la propiedad intelectual) tienen un valor económico y contribuyen al sostenimiento de las ventajas competitivas, por lo que su identificación y comunicación es una medida del desempeño estratégico (Bontis 2001). Los reportes de capital intelectual se construyen utilizando una serie de indicadores y métricas que dan testimonio de un conjunto de prácticas y factores "ocultos" que fundamentan la creación de valor (Edvinsson y Malone 1997). A un nivel más agregado, la mayoría de los estudios sobre medición y gestión de conocimiento o capital intelectual, construyen las variables relativas al conocimiento (los recursos 
intangibles o las capacidades) mediante un conjunto de ítems o afirmaciones donde los entrevistados expresan su grado de acuerdo en una escala de Likert (Bierly y Chakrabarti 1999, Lee et al.1999, Ordóñez de Pablos 2002, Choi y Lee 2003, Keskin 2005, Pai 2005).

Para el componente conceptual relativo al desempeño se han utilizado medidas financieras, tales como los retornos sobre inversiones, ventas, activos, etc. (Bierly y Chakrabarti 1999, Pai 2005). Pero está más generalizada la utilización de medidas indirectas, tales como la valoración sobre el cumplimiento de diversos objetivos (importancia para la empresa o con respecto a los competidores principales en diversas temáticas tales como éxito global, participación de mercado, tasa de crecimiento, rentabilidad, innovatividad $o$ ventaja competitiva relativa al diseño, calidad, servicio al cliente, reducción de costos, capacidad utilizada o mercados de exportación) (Desphande et al.1993, Katsikeas 1994, Drew 1997, Piercy et al. 1998, Moen 1999, Ordóñez de Pablos 2002, Keskin 2005, Schulz y Jobe 2001). La tabla 1 sistematiza un conjunto de enfoques al respecto.

Tabla 1. Efectos de la gestión del conocimiento sobre el desempeño.

\begin{tabular}{|c|c|c|c|}
\hline Autores & objeto de estudio & Variable de desempeño & Efecto \\
\hline $\begin{array}{c}\text { Bierly y } \\
\text { Chakrabarti } \\
(1996) \\
\text { Pai }(2005)\end{array}$ & $\begin{array}{l}\text { Clusters de empresas clasificados según el } \\
\text { foco del conocimiento: "explorador" } \\
\text { (interno) e "innovador" o "creativo" } \\
\text { (interno + externo) }\end{array}$ & $\begin{array}{l}\text { ROA } \\
\text { ROS } \\
\text { ROE }\end{array}$ & $\begin{array}{l}\text { Superior desempeño } \\
\text { comparativo }\end{array}$ \\
\hline $\begin{array}{l}\text { Choi y Lee } \\
\text { (2003) }\end{array}$ & Recursos humanos y sistemas integrados. & $\begin{array}{c}\text { Éxito global } \\
\text { Cuota de mercado } \\
\text { Tasa de crecimiento } \\
\text { Rentabilidad } \\
\text { Innovatividad } \\
\text { Tamaño }\end{array}$ & $\begin{array}{l}+ \\
+ \\
+ \\
+ \\
+ \\
+\end{array}$ \\
\hline Keskin (2005) & $\begin{array}{l}\text { Clusters de empresas clasificadas según su } \\
\text { estrategia orientada por lo explícito vs. la } \\
\text { estrategia orientada por lo tácito }\end{array}$ & $\begin{array}{c}\text { Éxito global } \\
\text { Cuota de mercado } \\
\text { Tasa de crecimiento } \\
\text { Rentabilidad } \\
\text { Innovatividad } \\
\text { Tamaño }\end{array}$ & $\begin{array}{l}\text { Desempeño superior } \\
\text { en las orientadas por } \\
\text { lo explícito }\end{array}$ \\
\hline $\begin{array}{l}\text { Schulz y Jobe } \\
\quad(2001)\end{array}$ & $\begin{array}{c}\text { Clusters de empresas clasificadas según su } \\
\text { estrategia: } \\
\text { orientada por lo tácito vs. estrategias } \\
\text { explícitas u orientadas por prácticas } \\
\text { internas y externas. }\end{array}$ & Desarrollo global & $\begin{array}{l}\text { Desempeño superior } \\
\text { en las orientadas por } \\
\text { lo tácito }\end{array}$ \\
\hline $\begin{array}{c}\text { Ordóñez de } \\
\text { Pablos (2002) }\end{array}$ & $\begin{array}{c}\text { Capital humano, relacional y estructural } \\
\text { Capital estructural }\end{array}$ & $\begin{array}{l}\text { Creación de valor } \\
\text { Desempeño } \\
\text { organizacional }\end{array}$ & $\begin{array}{c}\mathrm{NS} \\
+\end{array}$ \\
\hline $\begin{array}{l}\text { Ling-yee y } \\
\text { Ogunmokun } \\
(2001)\end{array}$ & Capital relacional y estructural & $\begin{array}{l}\text { Ventajas competitivas } \\
\text { exportadoras: } \\
\text { - en costos } \\
\text { - en } \\
\text { diferenciación } \\
\text { Desempeño exportador: } \\
\text { - metas } \\
\text { financieras } \\
\text { metas } \\
\text { estratégicas }\end{array}$ & $\begin{array}{l}+ \\
+ \\
+ \\
+\end{array}$ \\
\hline
\end{tabular}

Fuente: Elaboración propia con base en la revisión de literatura. 
Esta tabla muestra una corriente de literatura empírica que intenta describir la influencia de los recursos intangibles sobre el desempeño competitivo. La primera parte recoge una serie de estudios que, implícitamente, asocia estos recursos a las estrategias de gestión del conocimiento. Dichas estrategias se definen en función del foco del conocimiento (aspectos tácito comunicación directa o socialización- o explícito/codificado

documentación/uso de tecnologías de información) y su fuente de origen (desde el exterior -vía la adquisición o transferencia- o el interior -mediante prácticas de generación y distribución de conocimiento). Algunos autores sugieren que las empresas deben adaptar un enfoque excluyente $y$ orientarse únicamente hacia alguna estrategia (Hansen et al. 1999, Swan et al. 2000, Pai 2005) para tener efectos en el desempeño competitivo. La evidencia empírica obtenida por Keskin (2005) muestra que cada una tiene diferente impacto en el desempeño competitivo y que hay una jerarquía aparente (las empresas que desarrollan estrategias que combinan una orientación externa e interna muestran un desempeño superior con respecto a las que persiguen estrategias que combinan los enfoques tácito $\mathrm{y}$ explícitos, y estas últimas, también, son superiores a las que exclusivamente atienden a alguno de estos enfoques, y entre las que siguen un solo enfoque las que se guían por el aspecto tácito obtiene un mejor desempeño que las que tiene una aproximación explícita, aunque hay evidencia contradictoria, véase Schulz y Jobe, 2001). Sin embargo, otros autores manifiestan que las estrategias deben ser complementarias para obtener un mejor desempeño competitivo (Bierly y Chakrabarti 1999, Choi y Lee 2003, Zack 1996). Un estudio empírico reciente en empresas coreanas mostró que los modelos interno-tácito o externo-explícito pueden tener efectos sinérgicos mientras que la combinación tácita-explícita no es complementaria para obtener mejoras en el desempeño (Choi et al. 2006).

La segunda parte de la tabla muestra otros estudios (Ling-yee y Ogunmokun 2001, Ordóñez de Pablos 2002) que desarrollan empíricamente indicadores o proxies del capital intelectual y elaboran modelos multivariados que muestran su influencia sobre alguna medida de desempeño competitivo. Los resultados que obtienen revelan, en general, una influencia positiva de las diversas manifestaciones del capital intelectual sobre un buen número de indicadores de desempeño competitivo.

La evidencia empírica existente en la literatura no muestra claramente el efecto del capital intelectual sobre el desempeño. Dado que en general no se observan efectos sobre los indicadores financieros - o por lo menos en el corto plazo, se ha buscado más su efecto en las prácticas de creación de valor o en el desempeño competitivo. Parte del problema es que no se pueden dividir objetivamente, ni en lo conceptual ni en lo empírico, sus componentes. Independientemente de la aproximación o métodos para desagregar sus componentes -enfoque tácito o explícito, interno o externo, humano, estructural o relacional, el capital intelectual manifestado como prácticas tácitas o mediante soportes físicos ha mostrado efectos positivos -directos- en el desempeño organizacional.

En este documento se realiza un abordaje similar a lo reportado en la literatura: se construirán diversas medidas del capital intelectual y se indagará sobre su efecto en un conjunto de objetivos que representan el desempeño competitivo. Esperamos que las empresas que exploten sus recursos intangibles tengan mayores 
posibilidades de alcanzar sus objetivos competitivos y que la combinación de recursos incida selectivamente sobre los objetivos a alcanzar.

\section{METODOLOGÍA}

El estudio empírico se basa en una encuesta de la industria de maquinados industriales levantada en dos localidades mexicanas: Ciudad Juárez y Querétaro. El instrumento se aplicó entre 2005 y 2006 a 244 empresas de Querétaro $^{7}$ y 179 empresas de Ciudad Juárez $^{8}$. Este conjunto de empresas representa prácticamente el universo de las localidades. Se obtuvieron 179 y 104 respuestas válidas para este artículo respectivamente.

El cuestionario trata de cubrir diversas temáticas desde el perfil de la empresa y el propietario hasta sus estrategias, capacidades tecnológicas, actividades de aprendizaje aseguramiento de calidad e innovación, mecanismos de financiamiento hasta la valoración del entorno, las vinculaciones con otros agentes de la localidad y las políticas públicas. Se utilizaron preguntas cerradas de tipo cualitativo o categórico. También se incluyeron listados de recursos, actividades o productos para valorizar su importancia y frecuencia de implementación de acuerdo con una escala de Likert de cuatro puntos (donde $0=\sin$ importancia o nula frecuencia $\mathrm{y}$ $4=$ muy importante y muy frecuentemente). Todos los cuestionarios fueron levantados en entrevistas cara-a-cara.

\footnotetext{
${ }^{7}$ Encuesta a empresas de maquinados en Querétaro, UAM-X, 2005. Proyecto CONACYT-45550 "PYMES, redes de conocimiento, actividades innovativas y desarrollo local", UAM-X, UNAM, COLEF, UACJ, con la participación de la UdeG

${ }^{8}$ Encuesta a empresas de maquinados en Ciudad Juárez, UACJ, 2006. Proyecto CONACYT-45550 "PYMES, redes de conocimiento, actividades innovativas y desarrollo local", UAM-X, UNAM, COLEF, UACJ, con la participación de la UdeG
}

El sector de maquinados industriales de precisión está conformado por las empresas industriales que diseñan y fabrican piezas de precisión con niveles de 1 a 3 , de los 5 existentes en el sector a nivel internacional. Las empresas poseen equipos con diferente grado de complejidad y modernidad (manuales, $\mathrm{CN}$ o $\mathrm{CNC}$, con diferente cantidad de ejes). Las empresas son micro y pequeñas, el $43.2 \%$ en Ciudad Juárez y el 61\% en Querétaro cuentan con 6 o menos empleados, y sólo el $5.8 \%$ y el $7.3 \%$ de las empresas respectivamente tiene entre 30 y 70 empleados. La empresa más grande por empleo cuenta con 110 trabajadores.

Ambas localidades presentan marcadas diferencias en cuanto a la infraestructura técnico-productiva presente. Querétaro está ubicado en el centro del país y es un estado con alto dinamismo industrial que cuenta con una importante gama de agentes, tales como empresas, centros públicos de investigación y desarrollo, universidades, dependencias gubernamentales y asociaciones empresariales. Por otro lado, Ciudad Juárez está ubicada al norte del país y es frontera con Estados Unidos. Esta localidad presenta una fuerte demanda de productos maquinados por la industria maquiladora de exportación, pero no cuenta con una infraestructura tan rica como en el caso de Querétaro.

Como se describe con más detalle en las secciones siguientes, el análisis de la información se basó en un análisis multivariado para identificar grupos de prácticas empresariales que expresan diferentes recursos intangibles. Se estima su influencia sobre el desempeño competitivo a través de un conjunto de regresiones logísticas que asocian el cumplimiento de diversos objetivos innovadores a los recursos intangibles. 


\section{RESULTADOS}

\subsection{IDENTIFICACIÓN DEL CAPITAL INTELECTUAL}

Para identificar variables latentes, esto es, que no son fácilmente cuantificables ni imputables a una sola variable, se ha utilizado la técnica del análisis factorial tanto en estudios empíricos de capacidades tecnológicas como de recursos intangibles (DeCarolis y Deeds 1999, Ling-Yee y Ogunmokun 2001, Domínguez y Brown 2003, Vera-Cruz y Dutrénit 2005).

El conjunto original de factores expresa la información de 283 empresas con respecto a la siguiente información (11 variables):

1. Valoración de factores determinantes de competitividad (relativos a la calidad del producto y la capacidad de introducir innovaciones de la mano de obra) (2 variables)

2. Valoración de ventajas localizacionales (relativas a la disponibilidad de servicios técnicos) (1 variable).

3. Valoración de mecanismos de aprendizaje para crear $y$ compartir conocimiento (referentes a la capacitación y contratación del personal) (2 variables).

4. Frecuencia con que se realizan las actividades innovativas (relativas a la documentación de cambios de proceso y a la adaptación y modificación tecnológicas) (2 variables).

5. Frecuencia con que se realizan las actividades de aseguramiento de la calidad (relativas a la calibración y metrología, documentación de procesos de maquinado y mantenimiento de

$$
\begin{aligned}
& \begin{array}{l}
\text { maquinaria } \\
\text { variable). }
\end{array} \\
& \text { y equipo) }
\end{aligned}
$$

El análisis factorial permite reducir el número de variables a analizar dependiendo de sus correlaciones lineales. Así se obtienen subconjuntos de variables que están altamente correlacionadas en su interior, pero escasamente relacionadas con las de otros subconjuntos. Con el método de componentes principales se extraerá el nuevo espacio conformado por los subconjuntos que se denominan factores.

La solución de extracción propuesta se compone de cuatro ejes perpendiculares o factores, cada uno de los cuales está constituido por las combinaciones lineales de las 11 variables originales. Estos factores son los más importantes y representan el $60 \%$ de la variabilidad total de la muestra, como se observa en la Tabla 2.

La decisión sobre el número de factores a extraer ha estado influida por la construcción teórica del capital intelectual que señala tres subtipos claramente diferenciados. En este trabajo se seguirá el modelo de Bueno (2002) que subdivide al capital estructural en dos componentes: el capital organizacional y el tecnológico, con lo cual se tienen cuatro tipos de capital. Así el resultado de la extracción será más fácil de analizar pero hay que mantener ciertas reservas en cuanto a su fiabilidad. 
Tabla 2. Varianza Total Explicada

\begin{tabular}{|c|c|c|c|c|c|c|}
\hline \multirow[b]{2}{*}{ Componente } & \multicolumn{3}{|c|}{$\begin{array}{l}\text { Sumas de las saturaciones al cuadrado } \\
\text { de la extracción }\end{array}$} & \multicolumn{3}{|c|}{$\begin{array}{l}\text { Suma de las saturaciones al cuadrado de } \\
\text { la rotación }\end{array}$} \\
\hline & Total & $\begin{array}{c}\% \text { de la } \\
\text { varianza }\end{array}$ & $\%$ acumulado & Total & $\begin{array}{c}\% \text { de la } \\
\text { varianza }\end{array}$ & $\%$ acumulado \\
\hline 1 & 2.835 & 25.776 & 25.776 & 1.969 & 17.903 & 17.903 \\
\hline 2 & 1.674 & 15.214 & 40.991 & 1.747 & 15.884 & 33.787 \\
\hline 3 & 1.131 & 10.284 & 51.275 & 1.526 & 13.872 & 47.659 \\
\hline 4 & .937 & 8.520 & 59.795 & 1.335 & 12.137 & 59.795 \\
\hline
\end{tabular}

Método de extracción: Análisis de Componentes principales.

En el nuevo espacio factorial, el conjunto de variables originales muestra diversa calidad de representación (véase columna de comunalidades en tabla 3 ). Así tenemos un subconjunto de variables bien representadas en la solución (comunalidades $>0.65$ ) tales como la documentación de cambios de proceso, adaptación y modificación de tecnología adquirida, las reuniones de análisis y reflexión o la adopción de un programa de calibración y metrología. Otro conjunto de variables muestra una débil representación (comunalidades < 0.5 ), entre las que podemos mencionar la contratación de personal con mayor experiencia, la capacidad de introducir nuevos productos o procesos y la disponibilidad en la localidad de servicios técnicos especializados.

Tabla 3. Matriz de componentes rotada (a)

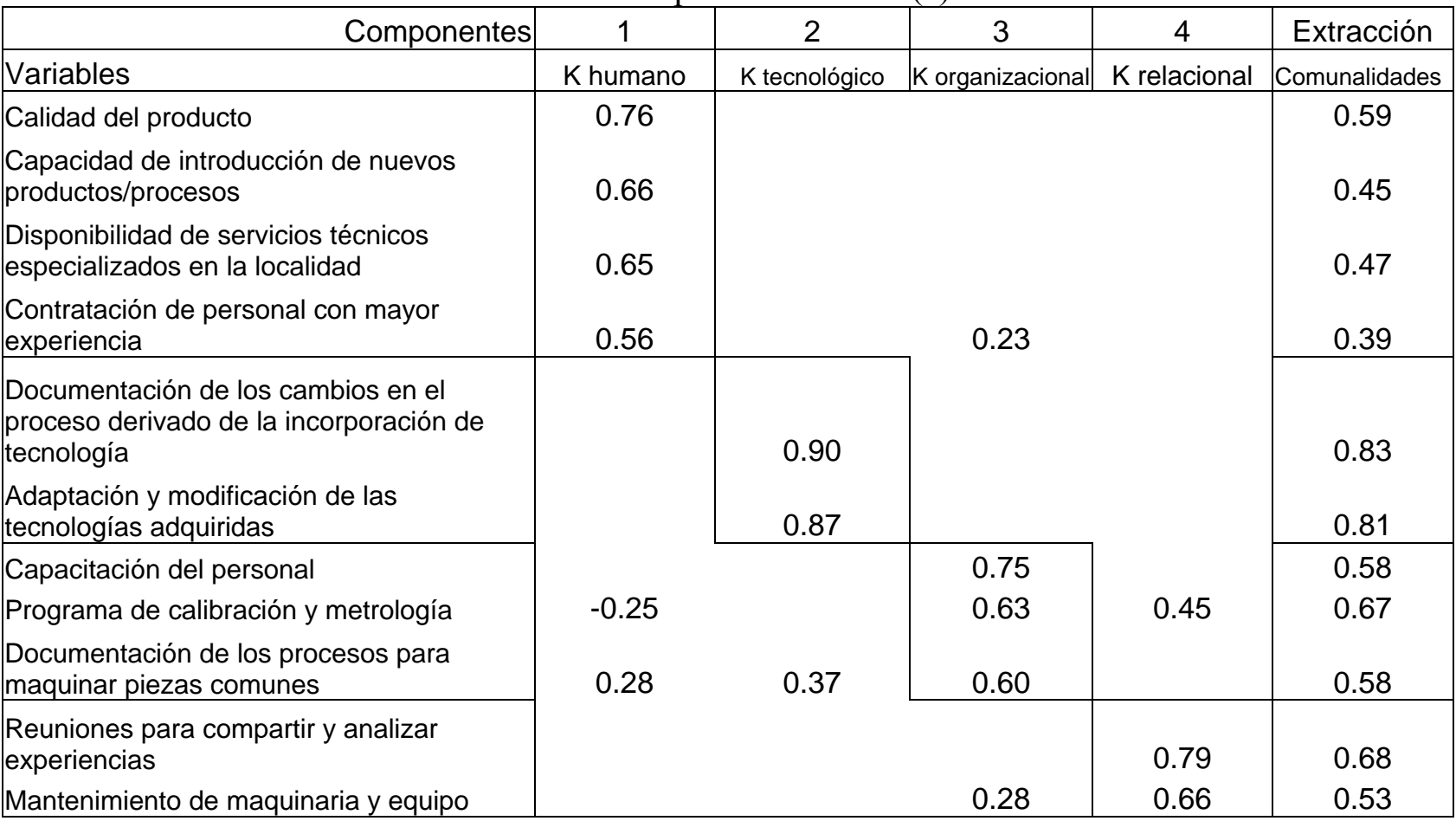

Método de extracción: Análisis de componentes principales. Método de rotación: Normalización Varimax con Kaiser.

(a) La rotación ha convergido en 5 iteraciones. 
El primer factor proyecta el $18 \%$ de la variabilidad de la muestra. Las variables que presentan una mayor saturación y tienen una mayor correlación entre sí son: las valoraciones sobre la calidad del producto y la capacidad de introducir nuevos productos/procesos como elementos de ventaja competitiva, la valoración de la ventaja localizacional asociada a la disponibilidad de servicios técnicos especializados y la contratación de personal experimentado como mecanismo de aprendizaje. El segundo factor explica casi el $16 \%$ de la variabilidad de la muestra. Está constituido por variables relativas a las actividades innovadoras. Saturan este factor la frecuencia con que se realizan la documentación de los cambios de proceso derivados de la incorporación de tecnología y la adaptación o modificación de tecnologías adquiridas. El tercer factor sólo contribuye con el $14 \%$ de la variabilidad de la muestra. De manera clara, presenta tres variables altamente correlacionadas entre sí y que son: la valoración de la capacitación del personal como mecanismo de aprendizaje, la adopción de un programa de metrología y calibración para asegurar la calidad, así como la documentación de procesos de maquinado. En el factor cuatro se agrupan un par de variables, cuya correlación contribuye a explicar el $12 \%$ de la varianza total. Se constituye con la práctica de las reuniones de análisis y socialización de experiencias $\mathrm{y}$ el mantenimiento de la maquinaria y equipo como medida para asegurar la calidad de la producción.

En resumen, en el primer factor tenemos variables intensivas en el know how del personal o asociadas a los recursos humanos tales como la habilidad innovativa, las habilidades de producción con calidad, el conocimiento técnico y la experiencia. Estas actividades pueden agruparse en la gestión del capital humano de la empresa. El segundo factor nos remite a las actividades innovativas orientadas a tener un mayor aprendizaje de fuentes externas de conocimiento, mediante la documentación de los procesos de producción, y a la utilización de la tecnología, vía la descodificación de know how incorporado en la maquinaria y equipo. Se puede, entonces, asociar a la gestión del capital tecnológico. En el tercer factor se combinan variables referidas a la gestión de rutinas y procedimientos para la capacitación, normalización y documentación de cambios realizados por la empresa. Se trata de actividades que pueden agruparse en la gestión del capital organizacional de la empresa dado que son procedimentales. Para finalizar, el cuarto factor se refiere a variables, que recogen prácticas de socialización del conocimiento y que son intensivas en relaciones interpersonales e intergrupales, como son las reuniones y los programas de mantenimiento de equipos. Se puede, entonces, asociar a la gestión del capital relacional, ya que por estas relaciones fluye información valiosa para la empresa.

Así en las pequeñas y medianas empresas del sector de maquinados industriales se pueden identificar prácticas de gestión del capital intelectual, tanto en su vertiente humana como en la tecnológica, organizacional y relacional. Cada empresa tiene una configuración diferente en su capital intelectual manifestado en las puntuaciones que presentan en cada uno de los cuatro factores. Con estas puntuaciones se puede asociar el capital intelectual a alguna medida de desempeño.

\subsection{EFECTOS SOBRE EL DESEMPEÑO COMPETITIVO}

El desempeño competitivo se puede medir, indirectamente, a través de la 
valoración de la importancia percibida sobre los objetivos de la actividad innovadora. Existe una correlación estrecha entre las valoraciones percibidas y los intentos estratégicos para alcanzar estos objetivos. En el gráfico 1 se observan las valoraciones sobre el impacto que tuvieron las innovaciones realizadas durante los últimos 5 años en el total de las empresas analizadas.

Esta serie de doce variables se han recodificado como 0 si el impacto sobre el objetivo estratégico no tuvo importancia o fue poco importante y 1 si el impacto fue importante o muy importante. La variable a predecir será la razón de las ventajas de haber otorgado una valoración importante con respecto a una baja valoración. La interpretación tiene que hacerse bajo el supuesto de que los objetivos valorados como importantes fueron conseguidos. Las variables explicativas de tal conducta serán los factores representativos del capital intelectual.
Gráfico 1. Valoración de objetivos innovadores.

\begin{tabular}{|l|}
\hline MMUY \\
IMPORTANTE \\
$\square$ IMPORTANTE \\
\\
IPOCO \\
IMPORTANTE \\
$\square$ SIN \\
IMPORTANCIA
\end{tabular}

Aumento participación externa

Regulaciones externas

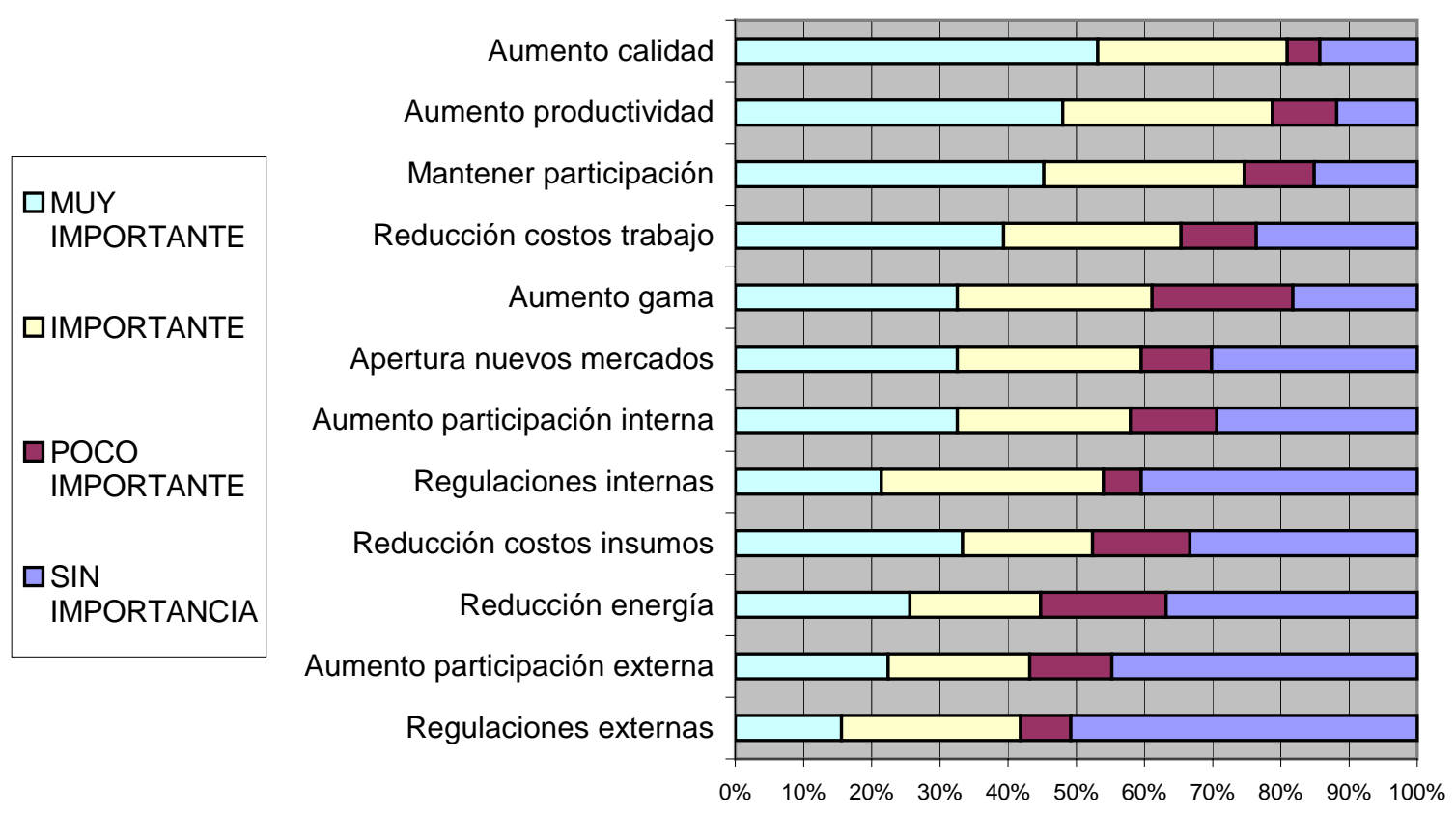

En las empresas estudiadas, al menos el $40 \%$ de la población calificó como importante o muy importante el conjunto de objetivos innovadores persiguiendo, prioritariamente, el aumento en la calidad de los productos, el aumento de la productividad en la empresa, el mantenimiento de la participación en los mercados, la reducción de los costos de trabajo y la ampliación en la gama de productos ofrecidos. Los objetivos menos valorados fueron los asociados a la internacionalización (como el cumplimiento con regulaciones externas y el aumento en la participación en el mercado externo) y la reducción en el consumo de energía.

\subsubsection{Las variables independientes y de control}

Las variables independientes se refieren a las puntuaciones obtenidas en el espacio factorial de dimensión 4 , donde cada variable representa un tipo de capital intelectual. Sus órdenes de magnitud se asocian a una valoración de 
la importancia o frecuencia de su implementación en las variables originales. Así los valores positivos se asocian a valoraciones altas y los valores negativos a valoraciones bajas. Por ejemplo, en el primer factor (capital humano), un cambio marginal positivo refleja un mejoramiento en la apreciación del subconjunto de las variables originales constituyentes de dicho factor.

La variable de control refleja los factores territoriales que se espera influyan sobre el desempeño innovativo. Intenta mostrar diferencias en la infraestructura y capacidad tecnológica regional. La variable de control es así la localización espacial. La probabilidad de alcanzar un objetivo innovador puede estar influida por aspectos regionales, por lo que se controla por la localización para evitar variaciones por diferencias de los dos sistemas locales de innovación. Se espera una diferente dotación de recursos y resultados empresariales, por ejemplo, inversión en capital fijo o el capital social, así como diverso acceso a los instrumentos financieros (públicos y privados) de fomento a la innovación que afecten el capital intelectual y la dirección y efectividad de los resultados innovadores.

\subsubsection{El modelo formal.}

Se utilizó un conjunto de regresiones logísticas para estimar una serie de modelos formales. Las variables dependientes son dicotómicas $(1=$ si el objetivo innovador fue conseguido y $0=$ si el objetivo no se consiguió) y las variables independientes son cuantitativas para los cuatro tipos de capital intelectual y categóricas para la variable de control, a saber LOCALIZACIÓN $(1=$ si la empresa está en Ciudad Juárez y $0=$ si está localizada en Querétaro).

La probabilidad de alcanzar un objetivo innovador viene dada por:

$$
\begin{aligned}
\mathrm{E}(Y i / X j i) & =\operatorname{Pr}(Y i=1 / X j i)=1 /\left[1+\mathrm{e}^{-}\right. \\
(\mathrm{z}) & +\varepsilon=\mathrm{e}^{(\mathrm{z})} / 1+\mathrm{e}^{(\mathrm{z})}+\varepsilon
\end{aligned}
$$

Siendo Z la combinación lineal:

$$
\begin{gathered}
\mathrm{Z}=\Sigma \beta \mathrm{j} X j \mathrm{ji}=\beta 1 \text { capital humano }+\beta 2 \\
\text { capital tecnológico }+\beta 3 \text { capital } \\
\text { organizacional }+\beta 4 \text { capital relacional }+ \\
\beta 5 \text { localización }+ \text { constante. }
\end{gathered}
$$

Mediante un conjunto de algoritmos diversos se obtuvieron combinaciones lineales de las variables independientes y de control que permiten estimar la probabilidad de que una empresa alcance un determinado objetivo innovador. Para los fines de este artículo nos interesa identificar las variables que tienen un efecto estadísticamente significativo sobre el desempeño innovador, así como su sentido. Para seleccionar las variables se utilizaron diversos métodos, desde la introducción en un solo paso hasta procedimientos por pasos (hacia atrás y hacia delante). De esta forma se obtiene información sobre la consistencia $y$ dirección de los efectos del capital intelectual sobre el desempeño innovador.

\subsubsection{Análisis de resultados}

La tabla 4 resume los resultados de la serie de regresiones. En la obtención de todos los objetivos innovadores, a excepción del cumplimiento de regulaciones externas, el capital humano tiene una influencia positiva sobre el desempeño. Los capitales tecnológico y relacional tienen una influencia más selectiva entre los objetivos innovadores. El capital organizacional parece no ejercer influencia alguna. 
Tabla 4. Efectos del capital intelectual sobre los objetivos innovadores.

\begin{tabular}{|c|c|c|c|c|c|c|}
\hline Variables dependientes & $\mathrm{K}$ humano & K tecnológico & K organizacional & $\mathrm{K}$ relacional & localización & MODELOS \\
\hline $\begin{array}{l}\text { 1) Aumento de } \\
\text { productividad en la } \\
\text { empresa }\end{array}$ & +++ & NS & NS & NS & NS & $\mathrm{M} 1, \mathrm{M} 2, \mathrm{M} 3$ \\
\hline $\begin{array}{l}\text { 2) Ampliación en la } \\
\text { gama de productos } \\
\text { ofrecidos }\end{array}$ & ++ & ++ & NS & NS & NS & M1,M2,M3 \\
\hline $\begin{array}{l}\text { 3) Aumento de la } \\
\text { calidad de productos }\end{array}$ & ++ & NS & NS & NS & NS & $\mathrm{M} 1, \mathrm{M} 2, \mathrm{M} 3$ \\
\hline $\begin{array}{l}\text { 4) Mantuvieron su } \\
\text { participación en los } \\
\text { mercados }\end{array}$ & +++ & ++ & NS & NS & NS & M1,M2,M3 \\
\hline $\begin{array}{l}\text { 5) Aumento de la } \\
\text { participación del mercado } \\
\text { interno de la empresa }\end{array}$ & ++ & NS & NS & NS & NS & M1,M2,M3 \\
\hline $\begin{array}{c}\text { 6) Aumento en la } \\
\text { participación del mercado } \\
\text { externo de la empresa }\end{array}$ & +++ & NS & NS & ++ & -- & $\mathrm{M} 1, \mathrm{M} 2, \mathrm{M} 3$ \\
\hline $\begin{array}{l}\text { 7) Permitieron que } \\
\text { la empresa abriera nuevos } \\
\text { mercados }\end{array}$ & ++ & NS & NS & NS & NS & M1,M2,M3 \\
\hline $\begin{array}{l}\text { 8) Permitieron la } \\
\text { reducción de costos de } \\
\text { trabajo }\end{array}$ & +++ & NS & NS & NS & NS & $\mathrm{M} 1, \mathrm{M} 2, \mathrm{M} 3$ \\
\hline $\begin{array}{l}\text { 9) Permitieron la } \\
\text { reducción de costos de } \\
\text { insumos }\end{array}$ & +++ & +++ & NS & NS & - & M2 \\
\hline $\begin{array}{l}\text { 10) Permitieron la } \\
\text { reducción de consumo de } \\
\text { energía }\end{array}$ & +++ & NS & NS & NS & -- & $\mathrm{M} 1, \mathrm{M} 2, \mathrm{M} 3$ \\
\hline $\begin{array}{l}\text { 11) } \quad \text { Permitieron } \\
\text { cumplir con regulaciones } \\
\text { del mercado interno }\end{array}$ & +++ & ++ & NS & + & NS & $\mathrm{M} 1, \mathrm{M} 2, \mathrm{M} 3$ \\
\hline $\begin{array}{l}\text { 12) } \quad \text { Permitieron } \\
\text { cumplir con regulaciones } \\
\text { del mercado externo }\end{array}$ & NS & + & - & NS & NS & M3 \\
\hline
\end{tabular}

Donde + Efecto positivo al $90 \%$ de significatividad, ++ Efecto positivo al $95 \%$ de significatividad, +++ Efecto positivo al $99 \%$ de significatividad, - Efecto negativo al $90 \%$ de significatividad, -- Efecto negativo al $95 \%$ de significatividad, NS No significativo. Las celdas en blanco indican que las variables no fueron seleccionadas por el método dado que no aportan información.

M1= Método por pasos hacia delante (Wald), M2= Introducción en un solo paso, M3 = Método por pasos hacia atrás.

Nuestros resultados muestran que el capital humano se revela como un insumo necesario para acometer cualquier objetivo innovador. Parece que no hay proceso de cambio o mejora sin la intervención de la habilidad, la experiencia y el conocimiento técnico de los recursos humanos. Este recurso resulta suficiente si lo que se pretende es un incremento en la productividad o en la calidad, o bien, una reducción en los costos del factor trabajo. Para alcanzar otros objetivos innovadores tales como la apertura de nuevos mercados y el aumento en la participación del mercado doméstico, nuestra regresión sugiere que el capital humano ha de combinarse con otro tipo de capital intelectual. La evidencia empírica en la literatura sugiere que objetivos asociados a la participación de mercado (Choi y Lee 2003) parecen más difíciles de acometer únicamente con el capital humano.

En nuestro análisis, el capital tecnológico en combinación con otros, en general con el capital humano -en cuatro de los cinco modelos-, influye sobre la consecución de objetivos relacionados con la política de productos, mercado, costos y regulaciones. Así, este capital parece apalancar a otros para lograr la diversificación, la supervivencia, la 
reducción de costos y el cumplimiento de la normatividad. Este resultado está en consonancia con lo reportado en la literatura (Choi y Lee 2003, Keskin 2005, Ling-yee y Ogunmokun 2001).

Con respecto al capital organizacional, la evidencia obtenida por nosotros no ratifica lo hallado en la literatura (Keskin 2005, Ordóñez de Pablos 2002, Ling-yee y Ogunmokun 2001) dado que los modelos de regresión no permiten demostrar su efecto en el cumplimiento de los objetivos innovadores. Sin embargo, si relajamos el nivel de significatividad de las pruebas estadísticas al $80 \%$, encontramos que tiene efectos mixtos: positivos, sobre el aumento de la productividad y la ampliación de la gama de productos, y negativos, sobre la participación de la empresa en el mercado doméstico así como en el cumplimiento de las regulaciones -tanto internas como externas. Sugerimos dos interpretaciones de este resultado: i) la apuesta por objetivos innovadores requiere como pre-condición el desarrollo de capital organizacional para poner a punto al capital humano (mediante la capacitación) y al capital tecnológico (mediante un programa de calibración y normatividad, así como prácticas de documentación de procesos de maquinado), y ii) el capital organizacional en estas empresas está escasamente desarrollado, lo cual limita e influye negativamente en sus estrategias competitivas para ganar mercado o incursionar en ámbitos foráneos.

En nuestros modelos, el capital relacional se asocia con tan sólo un par de objetivos innovadores: el incremento de la participación en mercados externos y el cumplimiento de regulaciones internas. De lo anterior existe evidencia parcial en la literatura, en particular, del efecto sobre el desarrollo global y la capacidad exportadora (Shulz y Jobe 2001, Lingyee y Ogunmokun 2001). Una explicación plausible para nuestros resultados es que en ambos objetivos se dibujan costos de transacción que pueden ser abatidos con una adecuada gestión del capital relacional. Si bien este tipo de capital parece pobremente expresado, habría que considerar que estas empresas están muy cerradas a la cooperación y vinculación, por lo que al parecer sus flujos de información tienen lugar en reuniones informales donde se comparten experiencias (al exterior, con los clientes básicamente) y después se socializan en las tareas de mantenimiento, donde es un requisito construir un ámbito cooperativo entre los trabajadores que realizan el servicio y quienes lo demandan.

En cuanto a la variable de control, la localización es estadísticamente significativa y de signo negativo en tres de los doce objetivos innovadores propuestos, esto es, las empresas localizadas en Querétaro tienen mayor probabilidad de cumplir con los siguientes objetivos innovadores: incremento de la participación en el mercado internacional, reducción de costos de trabajo y reducción del consumo de energéticos. Simplificando, se puede decir que el capital humano, expresado básicamente a través del aprendizaje individual, afecta directamente el cumplimiento de los objetivos innovadores en ambas localidades. Se trasluce, que quizás, los objetivos relacionados al costo de insumos, eficiencia energética e incremento en la participación en el mercado externo estén influidos por otro tipo de factores. Por ejemplo, de estímulos en la localidad para el abaratamiento de los insumos y reducir consumo energético o facilidades para relacionarse con agentes que promuevan la exportación (efecto positivo del capital relacional). 
Por otro lado, en la literatura empírica se hipotetiza que existen efectos indirectos -mediados por el capital estructural- de los capitales humano y relacional (Ordoñez de Pablos 2002). Esta situación se vislumbra en nuestro análisis de dos formas. De una parte, en el análisis factorial, el programa de calibración y la documentación de procesos (que constituyen el capital estructural, en su parte tecnológica y organizacional) participan de la saturación de los factores asociados al capital humano y relacional. Por otro, en nuestro análisis de regresión logística, el capital estructural parece ejercer alguna influencia sobre objetivos asociados al capital humano, como es el aumento de la productividad o la ampliación de la gama de productos. Entonces, es probable que las empresas persigan alcanzar objetivos innovadores en torno a su política de productos mediante estrategias, fundamentalmente, basadas en capital humano, aunque aquellas que busquen la diversificación, además, requieran del desarrollo del capital tecnológico, tal como se muestra en nuestro análisis.

Las estrategias para la gestión de la cuota de mercado también están afectadas por el capital humano. Para el mantenimiento de la participación parece existir cierta sinergia con el capital tecnológico, y para la ampliación en el mercado internacional con el capital relacional. El mensaje que puede desprenderse es que para sobrevivir (mantenimiento de la cuota de mercado) no basta la gestión del capital humano, se requiere desarrollar el capital tecnológico, y para intentar estrategias más agresivas -como arrebatar cuotas de mercado en el exterior- hay que invertir en capital relacional. Ling-yee y Ogunmokun (2003) demuestran que el capital relacional juega un papel único para las ventajas competitivas y el desempeño de empresas exportadoras.
En lo que se refiere a las estrategias de internacionalización, la decisión de incursionar en nuevos mercados parece estar influida por el capital humano, mientras que la de aumentar la cuota exportadora por el capital relacional. Aunque parece haber, también, alguna influencia de la localidad. Esta situación puede estar relacionada con los diferentes resultados e interpretaciones de los modelos explicativos de la probabilidad y propensión exportadora (Estrada 2005, Estrada y Heijs, 2006). Mientras que en los primeros las capacidades de aprendizaje e innovación tienen un claro efecto, en los segundos, es probable, que estas capacidades estén mediadas por el capital relacional. Entonces, para comenzar a exportar hay que ser una buena empresa (alto capital humano), pero para aumentar la cuota esto no es suficiente sino hay que tener buenas relaciones con el entorno (alto capital relacional) ${ }^{9}$.

La evidencia obtenida en este artículo sugiere que en la reducción de costos opera la gestión del capital humano. Además, se requiere que operen ciertas condiciones en la localidad para los insumos y energía. Así las variables relativas a los recursos humanos, en el primer factor, $\mathrm{y}$ las dotaciones territoriales o logísticas para los insumos, en el factor del entorno, inciden favorablemente en la disminución de costos. De aquí que una adecuada gestión del capital humano (p.ej. cultura de calidad e innovación, políticas de reclutamiento, outsourcing de servicios especializados o incentivos para retener personal experimentado) pudiera incidir favorablemente en una estrategia dirigida a reducir los costos.

\footnotetext{
9 La literatura sobre las ventajas competitivas nacionales sostiene que la estructura del sector constituye el contexto y es el principal factor determinante de los comportamientos de las empresas, los cuales, a su vez, explican los resultados obtenidos (Porter, 1980; Schmalensee, 1985).
} 
Para cumplir con las regulaciones, el capital intelectual parece manifestarse de múltiples formas. En relación a las exigencias requeridas al nivel nacional, los capitales humano, tecnológico y relacional parecen operar simultáneamente. En cuanto a los requisitos de los mercados internacionales, el capital intelectual parece tener tiene poco peso. Para acometer el objetivo de cumplimiento con las regulaciones internacionales un único modelo (de tres probados) fue significativo, en dicho modelo el capital humano no mostró influencia mientras que el tecnológico tuvo un efecto positivo y el organizacional uno negativo . La lectura interpretativa que se hace de este efecto negativo es que el mercado externo no demanda know how de piezas especiales (tanto de maquinado como de medición $y$ calibración) además de capacitación en temas selectos, que son, precisamente, las prácticas en que se está manifestando el capital organizativo en nuestro modelo.

\section{CONCLUSIONES}

La gestión del conocimiento propone que el conocimiento tácito de los empleados pueda ponerse al servicio de los objetivos de la empresa. En este artículo se presenta el caso de micro y pequeñas industrias de maquinados industriales en México. $\mathrm{Si}$ bien es cierto que no existe un esfuerzo sistemático por desarrollar la gestión, nuestra evidencia empírica permite identificar diversos conjuntos de prácticas intensivas en know how técnico, donde se deposita, comparte y transfiere conocimiento en un contexto muy específico. Tal como se planteaba en nuestro objetivo, estas prácticas expresan diferentes recursos intangibles que operacionalizamos como diversos componentes del capital intelectual. Los resultados de nuestros análisis muestran que existe una influencia estadísticamente significativa de este capital sobre el desempeño competitivo.

La evidencia presentada en este trabajo sobre las micro y pequeñas empresas de la industria del maquinado en México permite afirmar que en esta industria en las localidades analizadas el capital humano está asociado extensamente al cumplimiento de los objetivos estratégicos asociados al desempeño competitivo (i.e. ganancia en eficiencia, reducción de costos, cumplimiento de expectativas de los dueños, los clientes, proveedores o reguladores, etc), mientras que el capital tecnológico y el relacional tienen efectos selectivos. El capital tecnológico incide sobre diversas estrategias -producto, mercado, costos y normativas, y el capital relacional, únicamente, sobre las estrategias de internacionalización y normativas. Cabe destacar que el capital organizacional no muestra efectos significativos.

Estos resultados reafirman las propuestas de la administración basada en conocimiento, en particular al planteamiento que el conocimiento genera valor, los recursos intangibles son un medio para alcanzar ventajas competitivas y que no existe una sola combinación para obtener objetivos específicos (Prusak 1997, Edvinson y Malone 1997, enfoque de recursos y capacidades). También ratifica diversos hallazgos empíricos tales como la influencia del capital intelectual sobre el desempeño, al parecer existe alguna jerarquía entre sus diferentes componentes así como efectos mediadores y sinérgicos (Keskin 2005, Ordóñez de Pablos 2002). Sin embargo, también esta investigación manifiesta que continúa siendo un reto establecer sin ambigüedad sus componentes y que la jerarquía puede obedecer a contextos sectoriales y regionales específicos. Por 
ejemplo, la literatura destaca cierta prevalencia del capital estructural (Ordoñez de Pablos 2002, Ling-yee y Ogunmokun 2001), tanto en su parte de externalización (en soportes documentales o informáticas) como en su parte blanda (socialización, cultura rutinas, p.e. Schulz y Jobe 2001)., mientras que nuestra evidencia arroja que esta jerarquía en el contexto de pequeñas empresas (y/o el sector de maquinados industriales o ciertas regiones de México) está encabezada por el capital humano.

Se pueden identificar prácticas de gestión del capital intelectual en las PyMES de maquinados industriales en las localidades de Querétaro y Ciudad Juárez. El capital intelectual puede contribuir a la creación de valor y a sostener las ventajas competitivas en en el sector de maquinados dado que influye en el cumplimiento de los objetivos innovadores en ambas localidades. Se espera que el establecimiento de un sistema formal de gestión del conocimiento ayude a una mejor explotación de los recursos intangibles y pueda coadyuvar no sólo al mejoramiento del desempeño competitivo, sino, que mediante la dirección de la acumulación y focalización de recursos intangibles a la capacidad de diseño de estrategias, tanto al nivel de la empresa como de la región.

El cumplimiento de objetivos innovadores aparece influido de forma diferente por los recursos intangibles constituyentes del capital intelectual empresarial y por las condiciones del entorno. El capital humano, básicamente expresado a través de variables de aprendizaje tácito $\mathrm{e}$ individual, muestra un predominio sobre los otros tipos de capital y ejerce, persistentemente, una influencia positiva sobre la casi totalidad de objetivos innovadores.
Tanto en las empresas ubicadas en Querétaro como para la que se sitúan en Juárez, la política de productos (aumento en la productividad, la calidad y la gama) parece influida por el capital humano, aunque el objetivo innovador de diversificación, es probable, se vea más favorecido por el capital tecnológico.

La estrategia de internacionalización (mediante la apertura de nuevos mercados o vía el aumento en la participación), de las empresas de Ciudad Juárez y Querétaro, parece estar asociada a las habilidades, experiencia y cultura de los recursos humanos. Para aumentar la cuota exportadora, los flujos informales y las condiciones del entorno parecen interactuar más favorablemente en Querétaro. Estudios de la competitividad regional de estas dos ciudades muestran un mayor desarrollo institucional de Querétaro, con lo cual se podría explicar, en parte, la diferencias encontradas(Cabrero et al. 2005).

Las estrategias de reducción de costos, también dependen de la gestión del capital humano. En cuanto a costos laborales, no parece haber diferencia en empresas de Juárez y Querétaro, sin embargo en lo que se refiere a materias primas y reducción de costos energéticos, las condiciones de localización marcan distinciones por lo que sería interesante abundar sobre los condicionantes territoriales del capital humano y tecnológico en ambas localidades.

Finalmente, para el cumplimiento de regulaciones, el capital intelectual opera en múltiples formas dependiendo del mercado destino. En cualquier caso, hay que prestar atención a las habilidades para codificar y descodificar conocimientos de fuentes de abastecimiento tecnológico externo.

La interpretación de los resultados obtenidos en la industria de maquinados 
en dos localidades es una primera aproximación que difícilmente puede sostenerse con nuestros datos experimentales. Se recogen con el ánimo de provocar la reflexión y el debate tanto en el ámbito académico como en los de diseño de políticas y gestión pública. El ejercicio de caracterizar la gestión del conocimiento en un sector y localidad específicos demanda una profundidad $\mathrm{y}$ complejidad de análisis que rebasan este trabajo. En todo caso, nos encontramos frente a un fenómeno complejo que exige un esfuerzo sistemático para ir clarificando algunos elementos en torno a las prácticas, los recursos, las competencias, las estrategias, los aprendizajes, las interrelaciones y los desempeños en los sistemas locales de innovación. El esclarecimiento de estos factores demanda futuras investigaciones con diversos enfoques y abordes metodológicos.

\section{REFERENCIAS}

Amit, R.y Schoemaker, P.J.H. "Strategic Assets and Organizational Rent", Strategic Management Journal, vol. 14, 1993, pp. 33-46.

Barney, J. "Firms resources and sustained competitive advantage". Journal of Management, 17 (1), 1991, pp.99-120.

Bierly, P. y Chakrabarti, A."Generic Knowledge Strategies in the U.S. Pharmaceutical Industry", Strategic Management Journal, vol. 17, número especial de invierno, 1996, pp. 123-135.

Bontis, N. Assessing knowledge assets: a review of the models used to measure intellectual capital. International Journal of Management Reviews, 3(1), 2001, pp. 4061.

Bontis, N. The rising star of the chief knowledge officer, Ivey Business Journal, Vol.66, No.4, 2002, pp.20-25.

Bontis, N., Chong, W.C. and S. Richardson. "Intellectual Capital and the Nature of
Business in Malaysia", Journal of Intellectual Capital, 1 (1), 2000, 85-100.

Bontis, N., Crossan, M. and J. Hulland. "Managing an Organizational Learning System by Aligning Stocks and Flows", Journal of Management Studies, 39 (4), 2002, pp. 437-469.

Bueno, E "El capital social en el nuevo enfoque del capital intelectual de las organizaciones", Revista de Psicología del Trabajo y de las Organizaciones, Vol. 18, n. ${ }^{\circ}$ 2/3, 2002, pp. 157-176.

Bueno, E. y Morcillo, P. "Enfoques principales de dirección del conocimiento: una síntesis". Boletín Intellectus. IADEUniversidad Autónoma de Madrid.. No. 14, diciembre 2002, pp. 12-15.

Cabrera Izquierdo, A. y Rincón Hércules, M. "La Gestión del Conocimiento: Creando Competitividad en la Nueva Economía". Información Comercial Española. Nueva Economía y Empresa., 791, Abril-mayo 2001, pp. 77-91.

Cabrero Mendoza, Enrique, Alicia Ziccardi y Carlos Arce (coord.) (2005) Ciudades del siglo XXI: ¿Competitividad o cooperación?, Miguel Ángel PorrúaCámara de Diputados-CIDE, México,

2005.

Castells, M. The Rise of Network Society: Oxford. Blackwell Publishing, 2000.

Choi, B., Poon, S.K. y Davis, J.G. "Effects of knowledge management stategy on organizational performance: A complementary theory-based approach, Omega, doi: 10.1016/j.omega.2006.06.007, 2006.

Choi, B. y Lee, H. "An empirical investigation of KM styles and their effect on corporate performance". Information \& Management, 40, 2003, pp. 403-17.

Collis, D.J.; Montgomery C.A. "Competing on Resources: Strategy in the 1990s", Harvard Business Review, julio-agosto 1995, pp. 119-128.

Conolly, R.A.y Hirschey, M. "R\&D, Market Structure and Profits: A ValueBased Approach", Review of Economics and Statistics, vol. 66, 1984, pp. 682-686. 
DeCarolis, D.M.; Deeds, D.L. "The Impact of Stocks and Flows of Organizational Knowledge on Firm Performance: An Empirical Investigation on Biotechnology Industry", Strategic Management Journal, vol. 20, 1999, pp. 953-968.

Desphande, R. Jarley, U, Webster, F. "Corporate culture, costumer orientation and innovativeness in Japanese firms: a quadrad analysis". Journal of Marketing, 57, 1993, pp. 23-37.

Dierickx, I. y Cool, K. "Asset Stock Accumulation and Sustainability of Competitive Advantage", Management Science, vol. 35, 1989, pp. 1504-1511.

Domínguez, L y Brown, F. "Hacia una Propuesta de Medición de las Capacidades Tecnológicas de la Industria Mexicana". Ponencia presentada en $X$ Seminario Latino-Iberoamericano de Gestión Tecnológica. ALTEC 2003: "Conocimiento, Innovación y Competitividad: Los Desafíos de la Globalización. México, D.F. 22 a 24 de octubre 2003.

Dosi, G.: "Sources, Procedures, and Microeconomic Effects of Innovation", Journal of Economic Literature, vol. 26, 1988, pp. 1120-1171.

Drew, S. "From knowledge to action: the impact of benchmarking on organizational performance". Long Range Planning, 30 (3); 1997, pp. 427-41.

Estrada, S. Conducta tecnológica de la empresa y competitividad. Evidencia microeconómica para países en desarrollo: el caso de México. Tesis doctoral. Madrid, Universidad Autónoma de Madrid, 2005.

Estrada, S. y Heijs, J. "Technological behaviour and export probability in Developing Countries: The case of Mexico". Science, Technology and Society, Vol. 11, No. 2: 2006, pp.271-317.

Edvinsson, L. y Malone, M.S. Intellectual capital: realizing your company's trae value by finding its hiding brain power. New Cork, Harper Business, 1997.

García Muiña, F.E. y Navas López, J.E. El fenómeno tecnológico y su estudio en el pensamiento estratégico. Revista Madri $+\mathrm{d}$., No.23, Mayo-junio 2004.
Grant, R.M. "The Resource-based Theory of Competitive Advantage: Implications for Strategy Formulation", California Management Review, vol. 33, 1991, pp. 114-135.

Griliches, Z. "Market Value, R\&D and Patents", Economic Letters, no 7, 1981, pp. 183187.

Hansen, M., Nohria, N, Tierney, T. "What's your strategy for managing knowledge?". Harvard Business Review, 77 (2), 1999, pp. 106-16.

Hirschey, M.; Weygandt, J. J."Amortization Policy for Advertising and Research And Development Expenditures", Journal of Accounting Research, $\mathrm{n}^{\circ} 23,1985$, pp. 326335.

Itami, H.y Roehl, T. Mobilizing Invisible Assets, Cambridge, Harvard University Press, 1987.

Johnson, L. D. Pazderka, B. "Firm Value and Investment in R\&D", Managerial and Decision Economics, n ${ }^{\circ}$ 14, 1993, pp. 1524.

Katsikeas, C.S. "Export competitive advantages: the relevance of firms characteristics. International Marketing Review, 11(3), 1994, pp. 33-54.

Keskin, H. "The relationships between explicit and tacit oriented KM strategy and firm performance". Journal of American Academy of Business, 7 (1), 2005, pp. 16975.

Lee, H., Chang, Y. y Choi, B. "Analysis of effects of knowledge management strategies on corporate performance". Korea Intelligent Information Journal, 5 (2), 1999, pp. 99-120.

Ling-Yee, L. y Ogunmokun, G.O. "The influence of interfirm relational capabilities on export advantage and performance: an empirical analysis". International Business Review 10, 2001, pp. 399-420. www.elsevier.com/locate/ibusrev

Lynn, L.E. "The management of intellectual capital: the issues and the practice. Management Accounting Issues Paper 16, Management Accounting Practices Handbook. Hamilton, Society of Management Accountants of Canada, 1998. 
Moen, O. "The relationship between firm size, competitive advantages and export performance revisited" International Small Business Journal, 18 (1), 1999, pp. 53-72.

Ordóñez de Pablos, P. "Direct and Indirect Effects of Intellectual Capital on Organizational Competitive Advantage: Empirical Evidence". Paper presented at The Transparent Enterprise. The Value of Intangibles. Madrid. Noviembre 25-26, 2002.

Pai, D.C. "Knowledge strategies in Taiwan's design firms". Journal of American Academy of Business, 7 (2), 2005, pp. 73-7.

Pakes, A. "Patents, R \& D, and the stock market rate of return", Journal of Political Economy, vol. 93, 1985, pp. 390-409.

Peteraf, M.A. "The Cornerstones of Competitive Advantage: A Resource-Based View", Strategic Management Journal, vol. 14, 1993, pp. 179-191.

Piercy, N., Kaleta, A. y Katsikeas, C.S. "Sources of competitive advantages in high performing exporting companies". Journal of World Business, 33 (4), 1998, pp. 37893.

Pisano, G.P. "Knowledge, Integration, and the Focus of Learning: An Empirical Analysis of Process Development", Strategic Management Journal, vol. 15, 1994, pp. 85-100.

Porter, M.E. Competitive Strategy, New York, Free Press,.1980. Traducción al español: Estrategia Competitiva, México, C.E.C.S.A.,.1982.

Prusak, L. Knowledge in Organizations (Resources for the Knowledge-Based Economy) Newton, ButterworthHeinemann,. 1997.

Roos, G., Roos, J., Edvisson, L. y Dragonetti, N.C., Intellectual CapitalNavigating in the New Business Landscape, New York, New York University Press, 1998.

Sánchez, M.P., Chaminade, C. y Olea, M. "Management of intangibles: an attempt to build a theory, Journal of Intellectual Capital, Vol.1, no. 4. 2000, pp. 312-327.
Schmalensee, R. "Do Markets Differ Much?", American Economic Review, vol. 75, no. 3, 1985, pp. 341-351.

Shulz M. y Jobe LA. "Codification and tacitness as knowledge management strategies: an empirical exploration". Journal of High Technology Management Research, 12 (1), 2001, pp. 139-65.

Sveiby, K. E. The New Organizational Wealth: Managing \& Measuring Knowledge-Based Assets. San Francisco, Berrett-Koehler, 1997.

Swan J., Newell S, Robertson M. "Limits of IT-driven knowledge management for interactive innovation processes: towards a community-based approach", en Schriver B., Sprague RH (eds.) Hawaii International Conference on System Sciences, Los Alamitos, CA, Maui, HI: IEEE Computer Society Press, 2000.

Teng, B.S.; Cummings, J.L. "Trade-offs in Managing Resources and Capabilities", Academy of Management Executive, vol. $16, \mathrm{n}^{\mathrm{o}} 2$, 2002, pp. 81-91.

Vera-Cruz, A.O. y Dutrénit, G. Spillovers from MNCs through worker mobility and technological managerial capabilities of SMEs in Mexico. Innovation: management, policy \& practice. Vol 7, 2-3, April-august 2005, pp. 274-297.

Wernerfelt, B. "A resource based view of the firm" Strategic Management Journal, 5, 1984, pp. 171-180.

Zack, M.H."Managing Codified Knowledge". Sloan Management Review, 40 (4), 1999, pp. 45-58. 